\title{
Friction Estimation for Tendon-Driven Robotic Hands
}

\author{
Friedrich Lange, Martin Pfanne, Franz Steinmetz, Sebastian Wolf, and Freek Stulp
}

\begin{abstract}
In tendon-driven robotic hands, tendons are usually routed along several pulleys. The resulting friction is often substantial, and must therefore be modelled and estimated, for instance for accurate control and contact detection. Common approaches for friction estimation consider special dedicated setups, where the parameters of a static or dynamic friction model at a single contact point are determined. In this paper, we rather combine such individual friction models into an overall friction model for the entire finger. Furthermore, we propose a method for estimating the parameters of this overall model in situ, i.e. from trajectories executed on the assembled hand, avoiding the need for dedicated setups. An important component of the proposed model is a varying bias for treating friction at low velocities, allowing a simpler static friction model to be used. We demonstrate that our approach enables contacts to be detected more accurately on the DLR David hand, without additional sensors.
\end{abstract}

\section{INTRODUCTION}

Tendon-driven anthropomorphic robotic hands, such as the DLR David hand [1] in Fig. 1, are actuated by motors that are located in the forearm of the robot, rather than in the hand itself. This reduces finger size and increases dexterity. A disadvantage is the substantial friction that is caused by having to route tendons over multiple pulleys [2]. Additional friction may arise from tendons sliding over edges, especially at the extrema of the joint positions.

Modelling this friction is important for the motor control of tendon-driven hands, but also challenging. Specific friction effects at individual pulleys can be modelled well, and estimating their parameters is commonly done in dedicated identification setups [2]-[4]. But such setups do not take into account effects that arise only in the assembled hand. These include tendons sliding over edges, which depend on joint angles, or twisted mountings of the joints.

The first main contribution of this paper is to combine individual friction models into an overall friction model for the entire finger (described in Sect. IV). The second main contribution is to propose a method for estimating the parameters of this overall model in situ, i.e. from trajectories executed on the assembled hand (described in Sect. V). This makes parameter estimation not only more accurate - as it takes all friction effects that arise on the assembled hand into account - but also easier - as no dedicated hardware setups are required.

As it is difficult to separate time instants with motion from time instants with stiction in the assembled hand, our model

This work has been supported in part by the Hermann von HelmholtzGemeinschaft Deutscher Forschungszentren e. V. under Grant ZT-I-0010 (RedMod) and ZT-I-PF-5-20 (LearnGraspPhases).

The authors are with the Institute of Robotics and Mechatronics, German Aerospace Center (DLR), 82234 Wessling, Germany.

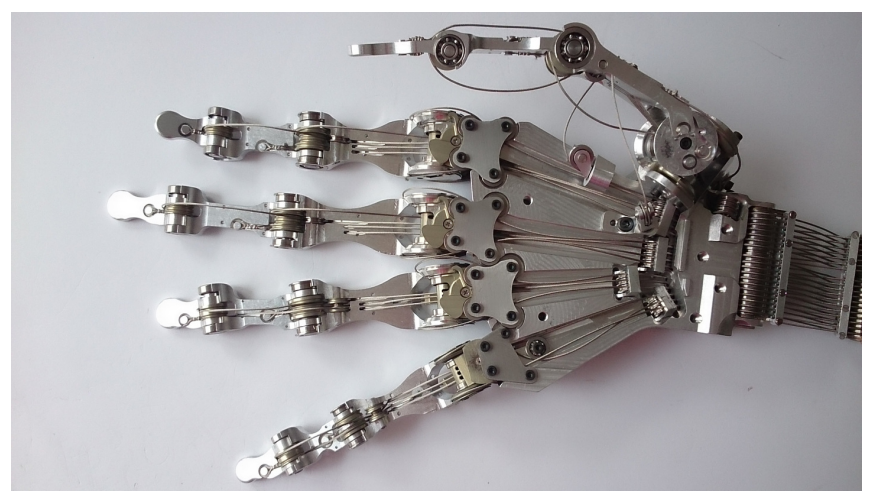

Fig. 1: DLR David hand (AWIWI II hand)

takes stiction into account explicitly. Our third contribution (described in Sect. VI) is to propose a varying bias for low velocities in combination with a static friction model. This eliminates the need for estimating the additional parameters of a dynamic friction model.

One of the detrimental consequences of the high (static) friction in tendon-driven hands is that it becomes hard to detect a contact - be it an intentional contact with an object that should be grasped, or an unintentional collision with an obstacle. Tactile sensors [5], [6] or proprioceptive sensors [7] can be used to detect such contacts, but they require further hardware integration and modification of the hand. A further contribution of this paper is to demonstrate (in the experimental section in Sect. VII) that our approach to friction modelling and estimation allows contacts to be detected on the DLR David hand, without any hardware modification. The approach can thus in principle also be applied to a wide variety of tendon-driven hands that do not have extra sensors for contact detection.

Before presenting the contributions in the sections mentioned above, we first present related work in Sect. II, and background on common friction models for individual sources of friction in Sect. III.

\section{ReLATED WORK}

Friction modeling has been developed predominantly for isolated joints [2], [3]. This is mainly because the friction of each tendon and joint can be measured separately in such setups, not only as the sum of multiple individual friction effects. A second reason is that recorded data can be separated in phases with static and dynamic friction, enabling the isolation of these effects. The parameters of a (simpler) static model are thus identified in isolation, by avoiding phases where the joint is in static (sticking) friction [8]-[10]. 
However static models are valid for constant speed only. In addition, they do not provide any information during stiction. The alternative are dynamic friction models ${ }^{1}$, having an internal state, such as the LuGre model [11]-[15].

Estimating a dynamic friction model is usually done with isolated joints, by first estimating the parameters of a static model from cyclic motion and evaluation of the parts with non-zero velocity only. The obtained parameters are then used as the base for the determination of a dynamic model [3].

In contrast to experiments with isolated joints, it can never be excluded that at least one tendon has zero velocity with respect to a single joint in the assembled hand. Therefore, static friction is always present in the measurements. Accurately estimating a static model thus fails, and its extension to a dynamic model is not possible.

Similar to this paper, the approach in [16] separates the effect of static and dynamic friction. However, the external force is assumed to be known and is thus taken as static friction force, which is not feasible for contact detection.

Recently, Ludovico et al. [4] proposed an extended Coulomb friction model for tendons which slide along an edge or into a bushing. They partition the space according to the sign of velocity and angle of diversion and model each part by the tendon tension and a sine function of the angle of diversion. In this way a parametric model is found.

Li et al. [17] designed a model for a passive arm with many joints and tendons, where the goal is to show equal curvature along the arm, once a torque is applied between two links. It turns out that this model strongly depends on friction. The setup assumes that all joints behave similarly, and the findings can thus not be transferred to a complex tendon-driven hand.

Other approaches consider hand models including friction [18], where the next state is predicted from the current state and the action with a data-driven method. The emphasis of these papers is on prediction of grasp states, less on contact torque measurements.

\section{Modelling Individual Friction EfFects}

In this section, we provide a summary of existing models for friction at individual pulleys and edges. In subsequent sections, these individual models are combined to construct friction models for complete tendon-driven fingers.

There are several static models to represent static and sliding friction similar to Fig. 2. They consist of three parts: 1) stiction forces $F_{s+}$ or $F_{s-}$ up to a breakaway force; 2) transient friction represented by Stribeck velocities $v_{s+}$ or $v_{s_{-}}$; 3) viscous friction for higher velocities, which is proportional to $F_{v+}$ or $F_{v-}$ with a base Coulomb friction $F_{c+}$ or $F_{c-}$ [11], [12]. For simplicity, $F_{s+}$ and $F_{s-}$ in Fig. 2 are replaced by $\pm F_{s}$ whenever symmetric values are assumed. This applies also for $F_{c}$ and $v_{s}$, but $F_{v-}=F_{v+}=F_{v}$.

\footnotetext{
${ }^{1}$ A static friction model allows only dynamic friction to be predicted, i.e. friction at (constant) non-zero velocity. A dynamic model is also able to represent static friction, i.e. friction at standstill.
}

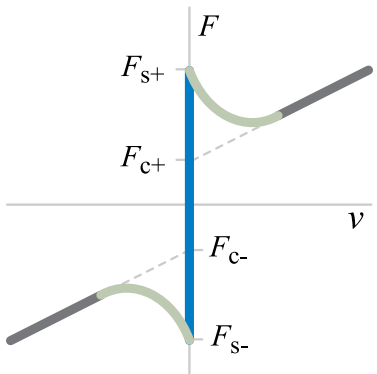

(a) Standard characteristic

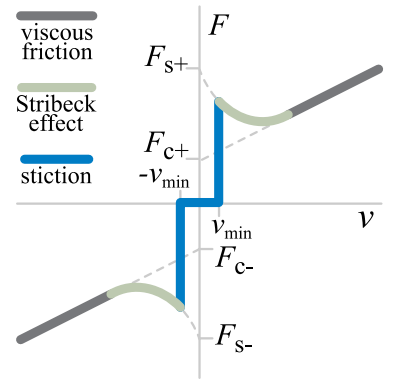

(b) Characteristic with $v_{\min }$, as proposed in Sect. VI
Fig. 2: Asymmetric static friction models. As to be described in Sect. IV, this figure is valid for both friction forces (where $v=\dot{x}$ ) and friction torques (where $F=\mathrm{T}$ and $v=\dot{q}$ ).

For a velocity $v \neq 0$ this is represented as

$$
F(v)=\left\{\begin{array}{ll}
F_{c+}+\left(F_{s+}-F_{c+}\right) e^{-v / v_{s+}}+F_{v+} v & \forall v>0 \\
F_{c-}+\left(F_{s-}-F_{c-}\right) e^{-v / v_{s-}}+F_{v-} v & \forall v<0
\end{array},\right.
$$

which is valid for both friction forces at tendons and friction torques at joints, with $v=\dot{x}$ or $v=\dot{q}$ respectively.

It should be noted that when used for friction within fingers, these parameters may depend on other variables, such as on the tendon force and the joint angle.

It is often assumed that friction at tendons is proportional to the tendon force (tension) [4], [19]. Thus the general dependence on the tendon force $f$ can be replaced by a multiplication, such that $F(f, v)=f F(v)$, with $F(v)$ computed by (1). Then the parameters of $F(v)$ depend at most on the joint angle. But such a dependence is assumed for fixed pulleys or edges only. There, friction depends non-linearly on the angle of diversion and thus on the joint angle [4]. In contrast, friction at pulleys with ball bearings has no further dependence.

\section{FRICTION IN TENDON-DRIVEN FINGERS}

In tendon-driven fingers, tendons are typically routed from the motors in the forearm through the wrist to the base of the hand. From there, they are routed through the finger, where the tendons to distal joints are routed through the more proximal joints, as can be seen in Fig. 1. Thus, there are multiple pulleys and edges along which friction arises. In this section, we first develop a model for the friction forces at the tendons (Sect. IV-A), then friction torques at the joints (Sect. IV-B), and finally the sum of these forces and torques (Sect. IV-C). Before doing so, we present the basic motion model for tendon-driven systems.

The basic equations of a tendon-driven systems with $m$ motors and $n$ joints are [20]:

$$
\begin{aligned}
\mathbf{x}_{1} & =\mathbf{R q} \\
\boldsymbol{\tau} & =\mathbf{R}^{\top} \mathbf{f}_{0} \\
\mathbf{f}_{0} & =\mathbf{K}\left(\mathbf{x}_{0}-\mathbf{x}_{1}\right) .
\end{aligned}
$$

They state that the joint side tendon position $\mathbf{x}_{1} \in \mathbb{R}^{m}$ (see Fig. 3) can be computed from the joint position (joint angle) $\mathbf{q} \in \mathbb{R}^{n}$ with the routing matrix $\mathbf{R} \in \mathbb{R}^{m \times n}$, and vice 
versa. The joint torque $\tau \in \mathbb{R}^{n}$ results from the individual tendon forces (tendon tensions) $\mathbf{f}_{0} \in \mathbb{R}^{m}$ using the transposed routing matrix. In the considered setup they are computed from the measured elongation $\mathbf{x}_{0}-\mathbf{x}_{1}$ of the tendons at the springs [21], assuming a known diagonal spring stiffness matrix $\mathbf{K} \in \mathbb{R}^{m \times m}$. The measured elongation in combination with the measured motor position and thus the motor side tendon position $\mathbf{x}_{0} \in \mathbb{R}^{m}$ yields $\mathbf{x}_{1}$ (see Fig. 3 ).

From (2), we obtain the relative motion for tendon $i$ at joint $j$ with respect to the link of joint $j-1$ with

$$
\dot{x}_{i j}=\sum_{j^{\prime}=j}^{n} r_{i j^{\prime}} \dot{q}_{j^{\prime}},
$$

where the $r_{i j}$ denote the elements of the routing matrix $\mathbf{R}$. In this paper, we will often drop $i$ to generalize over all tendons, e.g. $\dot{x}_{j}$ instead of $\dot{x}_{i j}$, or $r_{j}$ instead of $r_{i j}$.

In these equations, $\dot{x}_{j}$ is the relative velocity of a tendon within the link before joint $j$, i.e. with respect to the previous joint $j-1$. Thus $\dot{x}_{j}$ is the source of friction at pulley $j-1$ and at any edge between joints $j-1$ and $j$. In this way, $x_{1}$ is the tendon position before the first finger joint, i.e., the position at the joint side of the spring.

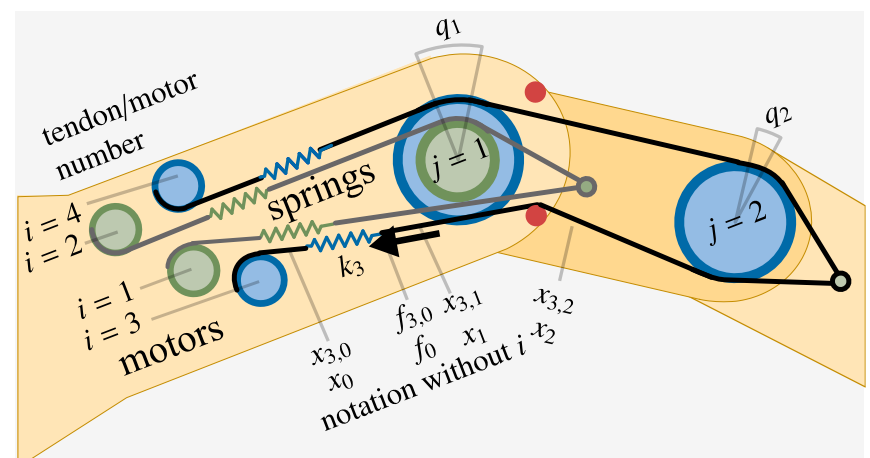

Fig. 3: Schematic illustration of a finger with two joints and four tendons, and the corresponding notation for tendon $i=3$. The tendon routing between the joints is limited by edges (marked as red spots).

\section{A. Friction forces at the tendons}

The friction at a tendon depends on the force acting along that tendon, and it differs at and between different pulleys in the finger. We propose a set of equations to iteratively estimate these forces from more proximal to more distal joints, based on the known tendon force at the spring, which is denoted $f_{0}$ (see Fig. 3).

For clarity, estimated friction forces are always capitalized $(F)$, whereas tendon forces are not $(f)$. Furthermore, we need to distinguish between forces at the pulley and between two pulleys, which are indicated with $A$ ('at') and $B$ ('between') respectively. Again, the $i$ index referring to the tendon number is dropped for clarity whenever possible. These forces and their notation are illustrated in Fig. 4.

With these definitions, the tendon forces before or at the individual joints, $f^{B}$ and $f^{A}$ respectively, see Fig. 4, are

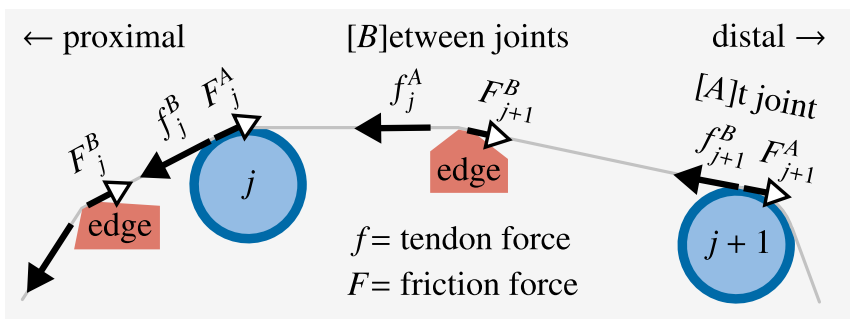

Fig. 4: Illustration of the tendon and friction forces along one tendon at joints $j$ and $j+1$.

defined as

$$
\begin{aligned}
& f_{j}^{B}=f_{0}-\sum_{j^{\prime}=1}^{j} f_{j^{\prime}-1}^{A} F_{j^{\prime}}^{B}\left(\dot{x}_{j^{\prime}}, q_{j^{\prime}-1}\right)-\sum_{j^{\prime}=1}^{j-1} f_{j^{\prime}}^{B} F_{j^{\prime}}^{A}\left(\dot{x}_{j^{\prime}+1}\right) \\
& f_{j}^{A}=f_{0}-\sum_{j^{\prime}=1}^{j} f_{j^{\prime}-1}^{A} F_{j^{\prime}}^{B}\left(\dot{x}_{j^{\prime}}, q_{j^{\prime}-1}\right)-\sum_{j^{\prime}=1}^{j} f_{j^{\prime}}^{B} F_{j^{\prime}}^{A}\left(\dot{x}_{j^{\prime}+1}\right),
\end{aligned}
$$

where $f_{0}$ is the measured tendon force at the spring, which is an element of $\mathbf{f}_{0}$ in (3).

Strictly speaking, the effect of each friction function $F_{j}^{A}$ or $F_{j}^{B}$ depends on the force at the respective pulley or edge at or before joint $j$. Instead, in order to make (6) and (7) unambiguous, we assume that friction depends on the force before the respective place, i.e. the effect of $F_{j}^{A}$ depends on $f_{j}^{B}$ and not on $f_{j}^{A}$ and that of $F_{j}^{B}$ on $f_{j-1}^{A}$ and not on $f_{j}^{B}$.

The functions $F_{j}^{B}$ and $F_{j}^{A}$ are instantiations of the individual friction functions $F$ described in Sect. III. The parameters of each function are simultaneously estimated for each tendon $i$ and each joint $j$ with the method described in Sect. V. For completeness, $q_{0}=0, f_{0}^{A}=f_{0}$, and $\dot{x}_{i, n+1}=0$ must also be defined to enable the sums to be computed.

\section{B. Friction torques at the joints}

In addition to the friction at the tendons, friction may also act on the joint itself, i.e. within the joint or at least independent from tendons. This friction torque $\mathrm{T}_{j}$ is modeled by a part $\mathrm{T}_{j v}$ depending on joint velocity and a part $\mathrm{T}_{j q}$ which only depends on the joint angle.

$\mathrm{T}_{j q}$ is the friction caused by the plastic cover of the finger. It is modeled as a torque which is linear with respect to the deviation from a built-in joint angle. So, strictly speaking, it is a rotational spring and not friction which would be modeled by (1).

$\mathrm{T}_{j v}$ represents the friction in the joint, and it is modeled with a twofold approach, i.e.,

$$
\mathrm{T}_{j v}\left(\sum_{i=1}^{m} f_{i j}^{B}, \dot{q}_{j}\right)=\mathrm{T}_{j v 0}\left(\dot{q}_{j}\right)+\mathrm{T}_{j v f}\left(\dot{q}_{j}\right) \sum_{i=1}^{m} f_{i j}^{B}
$$

with $\mathrm{T}_{j v 0}\left(\dot{q}_{j}\right)$ and $\mathrm{T}_{j v f}\left(\dot{q}_{j}\right)$ also being instantiations of $F$. Equation (8) includes joint friction that is independent of the acting force, e.g. at an almost jammed joint and joint friction which is proportional to the acting force, where the latter is summed up of all tendon forces. 


\section{Total friction torques}

The individual friction effects of Sects. IV-A and IV-B are concatenated to a complete equation whose parameters are estimated in Sect. V. In order to distinguish between the joint friction torque of Sect. IV-B, the torque caused by the friction forces of Sect. IV-A, and the total friction torque that effectively acts on joint $j$, we denote the latter by $\mathrm{T}_{j}^{\mathrm{eff}}$. The effective friction torque of joint $j$ is

$$
\mathrm{T}_{j}^{\mathrm{eff}}=\sum_{i=1}^{m} r_{i j} f_{i 0}-\tau_{j}^{\mathrm{ext}}=\tau_{j}-\tau_{j}^{\mathrm{ext}}
$$

with $\tau_{j}^{\text {ext }}$ being the torque that results in external forces and/or inertial forces of the finger. Note that with friction, $\tau_{j}^{\text {ext }}$ differs from $\tau_{j}$ in (3). $\mathrm{T}_{j}^{\mathrm{eff}}$ includes friction from all tendons and friction from the joint itself.Thus it can also be formulated as:

$$
\begin{aligned}
\mathrm{T}_{j}^{\mathrm{eff}} & =\sum_{i=1}^{m} r_{i j} \sum_{j^{\prime}=1}^{j} f_{i j^{\prime}-1}^{A} F_{i j^{\prime}}^{B}\left(\dot{x}_{i j^{\prime}}, q_{j^{\prime}-1}\right)+\sum_{i=1}^{m} r_{i j} \sum_{j^{\prime}=1}^{j} f_{i j^{\prime}}^{B} F_{i j^{\prime}}^{A}\left(\dot{x}_{i, j^{\prime}+1}\right) \\
& +\mathrm{T}_{j v 0}\left(\dot{q}_{j}\right)+\mathrm{T}_{j v f}\left(\dot{q}_{j}\right) \sum_{i=1}^{m} f_{i j}^{B}+\mathrm{T}_{j q}\left(q_{j}\right)
\end{aligned}
$$

where the effect of the friction forces at the tendons on the joint friction torques is represented analogously to (3).

\section{ESTIMATION OF THE FRICTION PARAMETERS}

Since the friction models of the individual fingers are not coupled, they are estimated independently from each other. Equations (10) and (1) represent the system for which the open friction parameters must be estimated for each finger, where the non-linear function (1) is linearized in each step of the estimation at the current working point $F_{s+}, F_{c+}, v_{s+}$, $F_{s-}, F_{c-}$, and $v_{s-}$. The resulting system is then linear in the parameters. Thus for every $j$ it can be expressed by

$$
\mathrm{T}_{j}^{\mathrm{eff}}=\psi_{j 0}+\boldsymbol{\psi}_{j}^{\top} \boldsymbol{\theta}
$$

with $\boldsymbol{\theta}$ being the vector of the parameters, including, e.g., $F_{v+}$ of $F_{j^{\prime}}^{A}$, and $\psi_{j}$ being a column of the matrix of coefficients $\boldsymbol{\Psi}$ with the respective element being $r_{i j} f_{j^{\prime}}^{B} \dot{x}_{i, j^{\prime}+1}$ if $\dot{x}_{i, j^{\prime}+1}>0$ and $j^{\prime} \leq j$, or 0 otherwise. $\psi_{j 0}$ makes sure that at the current working point the linearized equation (11) coincides with the sum of the effects of the non-linear friction laws (1).

To estimate the parameters, data is recorded during the execution of generated joint trajectories. This is done without external contact, as all measured torques (which are computed from the measured tendon forces) then arise from friction and inertial forces. The latter are understood here to comprise all forces caused by acceleration, rotation (centrifugal and Coriolis forces), and gravity. For controlled finger motion they can be neglected, because mass and inertia and the resulting inertial forces are very small relative to the contact and grasping forces. Thus, with (9) and $\tau_{j}^{\text {ext }}=0$, the left hand side of (11) can be replaced by

$$
\mathrm{T}_{j}^{\mathrm{eff}}=\sum_{i=1}^{m} r_{i j} f_{i 0}
$$

Then, the parameters $\boldsymbol{\theta}$ can be estimated using a least squares algorithm. Note that the parameters of all joints have to be estimated simultaneously, as some friction effects affect multiple joint torques. Therefore, a decoupled estimation is not possible.

Since the order of magnitude between joint values and tendon values or between $F_{c+}$ and $v_{s+}$ is different, an extended Kalman filter approach [22], [23] is used, which in contrast to a normal least squares algorithm allows the expected magnitude of each parameter to be specified. In addition, the non-linearity of (1) can be modeled as a timevariant system. Furthermore, this approach allows the time variance of additional parameters, which are introduced in the next section, to be explicitly specified.

The non-linear dependence of $F_{j^{\prime}}^{B}\left(\dot{x}_{i j^{\prime}}, q_{j^{\prime}-1}\right)$ in (10) on the joint angle $q_{j^{\prime}-1}$ is resolved by defining several working points for each joint and by linear interpolation between them. This is done since the approach of [4] would result in additional non-linearities which further complicate the estimation.

\section{TREATMENT OF STICTION}

As motivated above, static friction and a possible offset of the measured values have to be accounted for. It has already been mentioned in Sect. II that the extension of the static friction models (1) of each friction effect to the respective dynamical models, i.e. to models with internal states, is not feasible. Therefore, a new procedure has been developed.

The idea is that although the friction characteristics of Fig. 2a are not continuous, friction might be smooth with respect to the time. For continuous non-zero velocity this is obvious. Then static models (1) accurately represent the individual friction effects. Instead, for zero velocity we assume friction as time dependent parameters which we denote as slowly varying bias terms for every friction effect.

In order to be more realistic, we assume Fig. $2 b$ instead of Fig. 2a. This means that for $|v|<v_{\min }$, the effect of (1) is neglected and friction is represented exclusively by the (varying) bias term. The latter is initialized with the value of (1) when passing $|v|=v_{\min }$, since in this way smoothness is preserved. During the phase with $|v| \leq v_{\text {min }}$ the bias term has to track stiction, such that it coincides with (1), once $|v|=v_{\text {min }}$ is passed again. Then the bias term is reset to zero and (1) is used as long as $|v|>v_{\min }$.

There is no way for the adaptation of the individual bias terms in order to track the individual stiction effects. But a total bias term $b_{j}^{\text {eff }}$ of joint $j$ can be adapted, as long as there are no unknown external forces. Then the measured joint torque, which is computed from the measured tendon forces by (12), represents the sum of the friction effects with $|v|>v_{\text {min }}$ and of those of the bias terms.

This means that during training of the friction parameters, the vector of all parameters of the individual friction models is extended by $n$ additional variables, the bias terms $b_{j}^{\text {eff }}$. These variables are assumed to have a significant change, i.e., in contrast to the other parameters their estimation is considered to be significantly time-dependent. However, 
a too big assumption of the time-variance will result in very small compensated torques, i.e. the complete friction model represents the measured joint torques (12) to a large extend, almost independent from the parameters. Instead, time-variance has to be designed in such a way that the bias terms represent obvious offset terms, but contact can still be detected by a significant mismatch between the modeled friction and the measured torques. ${ }^{2}$

On the other side, after the training, the prediction of the current friction is not only a computation from (10) with (1) and the estimated parameters. Even omitting the bias terms is not expedient. Instead, the bias that acts during prediction has to be estimated. This can be done by simply filtering the compensated torques or by $n$ further Kalman filters with a single estimated value each and the assumptions on timevariance taken from the training phase. Too big assumptions here as well result in missing to detect contact that could be seen from slowly increasing external torques. During prediction, the complete update of the bias is inhibited whenever a contact is detected, i.e., whenever a compensated torque exceeds a threshold.

So the characteristic of Fig. $2 b$ instead of $2 a$ is used for both training and prediction. In this way, a velocity threshold is defined below which friction is considered as stiction. During training, such time steps are not included in the estimation of the respective friction parameters.

For the bias the following rules apply at each friction effect, see also Fig. 5: When moving from $\left|v\left(t_{1}-1\right)\right|>v_{\min }$ to $\left|v\left(t_{1}\right)\right| \leq v_{\min }, b_{j}$ and $F\left(v\left(t_{1}\right)\right)$ are set to $F\left(\operatorname{sgn}\left(v\left(t_{1}-1\right)\right) v_{\min }\right)$ and zero, respectively, where $t_{1}$ is the first time step with $|v| \leq v_{\text {min }}$. During $|v| \leq v_{\text {min }}$, slow changes of $b_{j}$ are assumed, represented by a time-variant approach for the estimation of the bias. When moving from $|v(t-1)| \leq v_{\text {min }}$ to $|v(t)|>v_{\text {min }}$, $b_{j}$ is set to $-F\left(\operatorname{sgn}(v(t)) v_{\min }\right)$ and $F(v(t))$ is used again, where $t>t_{1}$ is the current time step. In addition, the difference between $F\left(\operatorname{sgn}\left(v\left(t_{1}-1\right)\right) v_{\min }\right)$ and $F\left(\operatorname{sgn}(v(t)) v_{\min }\right)$ is subtracted, decreasing for a longer stay at $|v| \leq v_{\min }$. Finally, during contact at $|v| \leq v_{\min }, t_{1}$ is set to $t$.

This has the following effects: There is no step when moving around $v=v_{\min }$ or $v=-v_{\min }$. For fast zero crossing of the velocity the behavior is identical with and without an intermediate sampling step with $|v| \leq v_{\min }$. This means that the change of the bias when entering the range with $|v| \leq v_{\min }$ is undone when leaving it. For a longer stay at $|v| \leq v_{\min }$, the previous change of the bias is forgotten. Instead, it is assumed that the slowly changed bias is appropriate when leaving the range with $|v| \leq v_{\min }$. If a zero crossing happens during contact, it is treated as fast zero crossing, i.e. the bias is not modified permanently.

These individual updates of the bias then result in the effective bias $b_{j}^{\text {eff }}$ of joint $j$, which is initialized by $\tau_{j}$ at the beginning, i.e. with the system at rest and without contact. Then it is updated with the effects of all biases $b$ which are set in the respective time step. These biases are denoted as

\footnotetext{
${ }^{2}$ In contrast to the prediction phase, during training the setup guarantees that no external forces are effective.
}

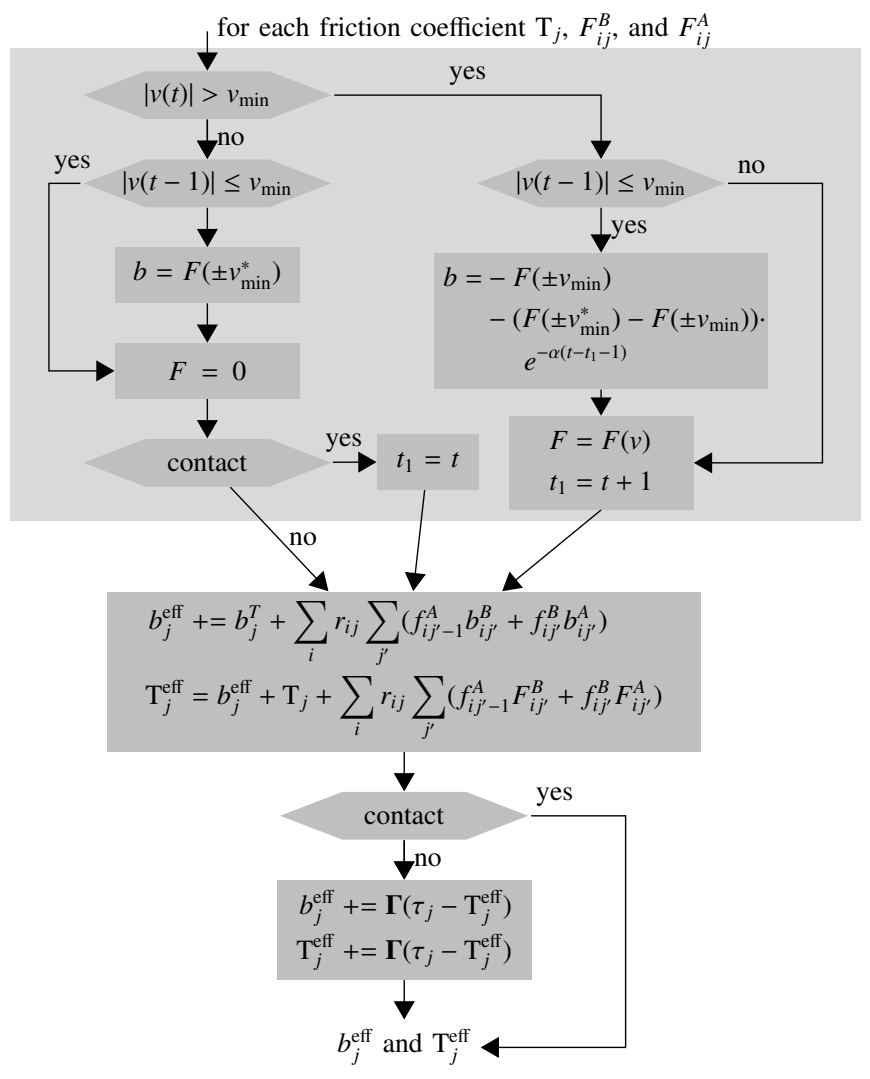

Fig. 5: Procedure for computing the bias.

$b_{j}^{T}, b_{i j}^{B}$, and $b_{i j}^{A}$, analogously to the corresponding friction functions $\mathrm{T}_{j}, F_{i j}^{B}$, and $F_{i j}^{A}$.

\section{EXPERIMENTS}

Experiments are performed with the index finger of the DLR David hand [1], depicted in Fig. 1.

For training, sample trajectories of $400 \mathrm{~s}$ duration are generated, in which all joints of a single finger are moved at the same time, but with different velocities. The parameters are estimated using the approaches of Sects. V and VI, with recorded tendon forces and motor positions from a single trajectory with low pretension of the tendons, i.e., low stiffness of the tendon-driven system. For evaluation, this trajectory is then repeated where from time to time a force is exerted on the finger tip. $v_{\min }=0.0005 \mathrm{~m} / \mathrm{s}$ and $v_{\text {min }}=0.01 \mathrm{rad} / \mathrm{s}$ are used respectively. The change of the bias in Sect. VI is considered by a variance of the changes of $10^{-9}$ at a variance of the assumed noise of $10^{-4}$, both being configuration parameters of the Kalman filter [22].

It turns out that the training converges better whenever $1 / v_{s}$ is used as a parameter instead of $v_{s}$ since in this way there is no singularity. Furthermore, $v_{s}$ is limited to $v_{s}>v_{s_{\min }}$ ( $v_{s+}$ and $v_{s-}$ accordingly) since the exponential function may exceed the stability region of the estimation whenever its argument becomes positive. In addition, the variance of the assumed noise for the Kalman filter estimation and the threshold for contact detection are increased for higher pretension. Finally, $f_{i j}$ is checked for $f_{i j}<0$. This means that 


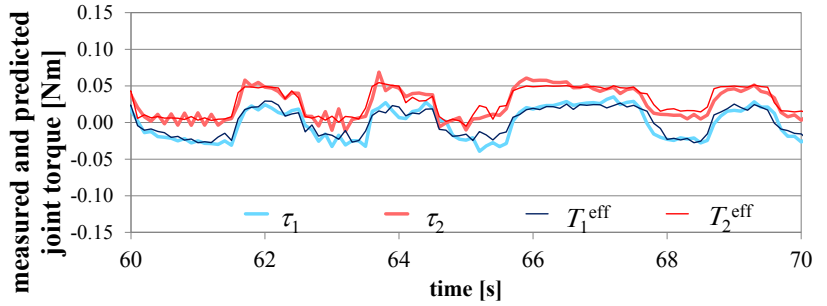

Fig. 6: Joint torques computed from the measured tendon forces and predicted joint torques with low pretension and no external forces.

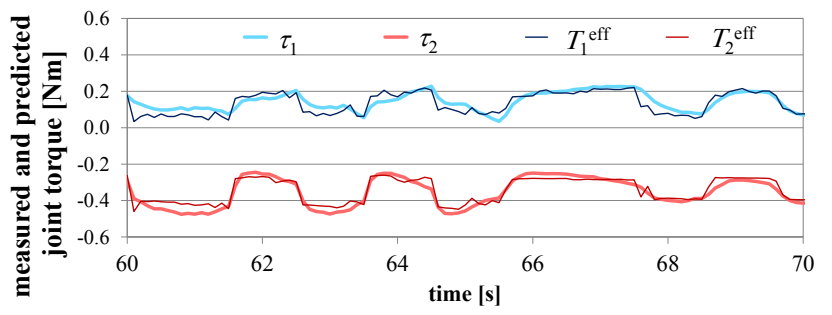

Fig. 7: Joint torques computed from the measured tendon forces and predicted joint torques with four times as high pretension and no external forces.

the friction forces of this tendon exceed the total tendon force $f_{i}$. The training should be modified whenever this appears.

\section{A. Results}

Figs. 6 and 7 show sections of training trajectories with different pretensions. In each case, the first two joint values are displayed of both the predicted friction torques and those that are computed from the measured tendon forces. The figures show that the trained model represents the friction torques also with untrained pretension.

\section{B. Discussion}

It turns out that a symmetric friction characteristic is adequate. This reduces the number of parameters including the bias from 820 to 472 . Furthermore, the first two joints are sufficient for detecting a contact force from any direction.

The uncompensated data of Fig. 7 show significant timevarying offsets which cannot be explained by the reported friction models for the hand. They are probably caused by static friction in the wrist. The proposed approach can compensate for it, in contrast to all of our previous attempts without the assumption of a bias.

As explained, it is trivial to reach small compensated torques by assuming a big time variance for the bias. Fig. 8 therefore displays a test in which external forces are exerted, measured by a force sensor. Modeling is quite accurate such that in periods with external force, the estimated external torques, i.e. the differences between the measured torques $\tau_{j}$ and the predicted torques $\mathrm{T}_{j}^{\text {eff }}$ far exceed the modeling error. A quantitative evaluation is however not possible, as neither the contact point nor the direction of force are known.

External forces should cause an external torque on joint 1 or 2. However the small torques close to $t=78 \mathrm{~s}$ exhibit that a substantial component has been exerted perpendicular to
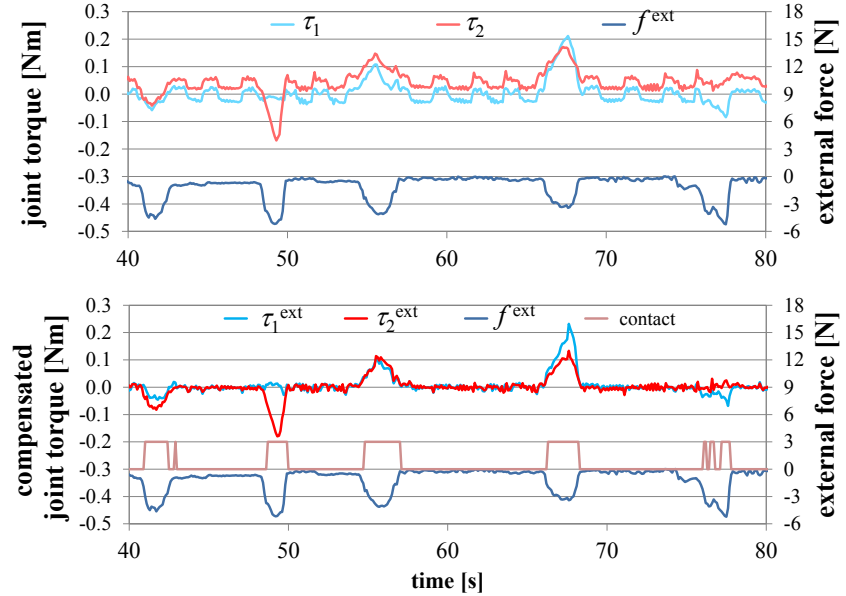

Fig. 8: Joint torques computed from the measured tendon forces (above) and compensated joint torques (below) during motion, sensed external force (absolute value) and resulting contact detection.

both such that the force causes almost no joint torques. This is a limitation of using joint torques for contact detection.

Furthermore, one may suppose that only those contacts can be detected that cause joint torques superior to the static friction torques. This is supported by the fact that during the stiction phase it is not possible to distinguish between static friction and an external torque not causing motion. So the uncertainty of the effective torque would sum up from $F_{s+}$ $F_{s-}$ of all involved friction effects, multiplied with the pulley radius and the tendon force. Instead, Fig. 8 shows that most contacts are detected though the compensated torques are not much more than the offset of $\tau_{2}$. This might be explained by the assumption that any change of the measured tendon forces is due to an external torque, unless it is caused by motion.

The estimated friction model is intended primarily for contact detection. Its use for quantitative measurements cannot not be validated with the current setup, which allows training only for zero torque or small torques caused by inertial or gravitational effects. Though the tendon force measurements are calibrated, it is not assured that this model will extrapolate correctly for significant torques.

\section{Conclusion}

In this paper, we have presented a novel approach to the modeling of friction in tendon-driven fingers, and the in situ estimation of the parameters of the model from trajectories executed on the assembled hand. This avoids the need for special setups or disassembly of the fingers for parameter estimation. Identifying the parameters of a dynamic friction model is very challenging for assembled hands. Instead, we have proposed a static model with bias terms added to the parametric model, to take into account friction at all places where tendons or joints may slide. These bias terms are also estimated. With this model, we demonstrate that it is possible to detect external forces, even if their effect does not exceed the level of disturbances of the joint torques. 


\section{REFERENCES}

[1] W. Friedl, M. Chalon, J. Reinecke, and M. Grebenstein, "FRCEF: the new friction reduced and coupling enhanced finger for the awiwi hand," in Proc. 2015 IEEE/RAS Int. Conf. on Humanoid Robots (HUMANOIDS), Seoul, Korea, Nov 2015, pp. 140-147.

[2] G. Palli and C. Melchiorri, "Friction compensation techniques for tendon-driven robotic hands," Mechatronics, vol. 24, pp. 108-117, Mar 2014.

[3] M. Iskandar and S. Wolf, "Dynamic friction model with thermal and load dependency: modeling, compensation, and external force estimation," in Proc. 2019 IEEE Int. Conf. on Robotics and Automation (ICRA), Montreal, Canada, May 2019, pp. 7367-7373.

[4] D. Ludovico, P. Guardiani, A. Pistone, J. Lee, F. Cannella, D. G. Caldwell, and C. Canali, "Modeling cable-driven joint dynamics and friction: a bond-graph approach," in Proc. 2020 IEEE/RSJ Int. Conf. on Intelligent Robots and Systems (IROS), Las Vegas, NV, USA (virtual), Oct 2020, pp. 7285-7291.

[5] J. R. Guadarrama-Olvera, E. Dean-Leon, F. Bergner, and G. Cheng, "Pressure-driven body compliance using robot skin," in Proc. 2019 IEEE/RSJ Int. Conf. on Intelligent Robots and Systems (IROS), Macau, China, Nov 2019.

[6] A. Vazhapilli Sureshbabu, M. Maggiali, G. Metta, and A. Parmiggiani, "Design of a force sensing hand for the R1 humanoid robot," in Proc. 2017 IEEE/RAS Int. Conf. on Humanoid Robots (HUMANOIDS), Birmingham, UK, Nov 2017, pp. 703-709.

[7] A. Zwiener, R. Hanten, C. Schulz, and A. Zell, "ARMCL: ARM contact point localization via monte carlo localization," in Proc. 2019 IEEE/RSJ Int. Conf. on Intelligent Robots and Systems (IROS), Macau, China, Nov 2019, pp. 7105-7111.

[8] A. C. Bittencourt and S. Gunnarsson, "Static friction in a robot joint - modeling and identification of load and temperature effects," ASME Journal of Dynamic Systems, Measurement, and Control, vol. 134, no. $5,2012$.

[9] M. Indri, I. Lazzero, A. Antoniazza, and A. M. Bottero, "Friction modeling and identification for industrial manipulators," in Proc. 18th IEEE Int. Conference on Emerging Technologies E Factory Automation (ETFA 2013), Cagliari, Italy, 2013, pp. 99-108.

[10] A. Wahrburg, S. Klose, D. Clever, T. Groth, S. Moberg, J. Styrud, and H. Ding, "Modeling speed-, load-, and position-dependent friction effects in strain wave gears," in Proc. 2018 IEEE Int. Conf. on Robotics and Automation (ICRA), Brisbane, Australia, May 2018, pp. 20952102.

[11] H. Olsson, K. J. Åstrom, C. Canudas de Wit, M. Gafvert, and
P. Lischinsky, "Friction models and friction compensation," European J. of Control, vol. 4, no. 3, pp. 176-195, Dec. 1998.

[12] Y.-H. Sun, T. Chen, C. Q. Wu, and C. Shafai, "Comparison of four friction models: Feature prediction," J. of Computational and Nonlinear Dynamics, vol. 11, no. 3, Oct. 2015.

[13] C. Canudas de Wit, H. Olsson, K. J. Åstrom, and P. Lischinsky, "A new model for control of systems with friction," IEEE Trans. Autom. Control, vol. 40, no. 3, pp. 419 - 425, Mar. 1995.

[14] K. J. Åstrom and C. Canudas de Wit, "Revisiting the LuGre friction model," IEEE Control Systems Magazine, vol. 28, no. 6, pp. 101-114, 2008.

[15] F. Al-Bender, V. Lampaert, and J. Swevers, "The generalized Maxwellslip model: A novel model for friction simulation and compensation," IEEE Trans. on Automatic Control, vol. 50, no. 11, pp. 1883-1887, Nov. 2005.

[16] D. Karnopp, "Computer simulation of stick-slip friction in mechanical dynamic systems," Journal of dynamic systems, measurement, and control, vol. 107(1), no. 1, pp. 100-103, 1985.

[17] Y. Li, Y. Liu, D. Meng, X. Wang, and B. Liang, "Modeling and experimental verification of a cable-constrained synchronous rotating mechanism considering friction effect," in Proc. 2020 IEEE/RSJ Int Conf. on Intelligent Robots and Systems (IROS), Las Vegas, NV, USA (virtual), Oct 2020

[18] A. Sintov, A. Morgan, A. Kimmel, A. Dollar, K. Bekris, and A. Boularias, "Learning a state transition model of an underactuated adaptive hand," IEEE Robotics and Automation Letters (RA-L), vol. 4, no. 2, pp. 1287-1294, 2019.

[19] J. Reinecke, M. Chalon, W. Friedl, and M. Grebenstein, "Guiding effects and friction modeling for tendon driven systems," in Proc. 2014 IEEE Int. Conf. on Robotics and Automation (ICRA), Hong Kong, China, May/June 2014, pp. 6726-6732.

[20] H. Kobayashi, K. Hyodo, and D. Ogane, "On tendon-driven robotic mechanisms with redundant tendons," The Int. Journal of Robotic Reasearch (IJRR), vol. 17, no. 5, pp. 561-571, May 1998.

[21] W. Friedl, M. Chalon, J. Reinecke, and M. Grebenstein, "FAS: a fexible antagonistic spring element for a high performance over actuated hand," in Proc. 2011 IEEE/RSJ Int. Conf. on Intelligent Robots and Systems (IROS), San Francisco, CA, USA, Sep 2011, pp 1366-1372.

[22] P. S. Maybeck, Stochastic Models, Estimation, and Control, ser. Mathematics in Science and Engineering. 141-1. Academic Press, New York, 1979, iSBN 978-0-12-480701-3.

[23] R. E. Kalman, "A new approach to linear filtering and prediction problems," J. Basic Eng., vol. 82, no. 1, pp. 35-45, 1960. 